W. Klingenberg

Nagoya Math. J.

Vol. 69 (1978), 107-116

\title{
ÜBER DEN INDEX GESCHLOSSENER GEODÄTISCHER AUF FLÄCHEN
}

\author{
WILHELM KLINGENBERG*
}

1. Wir betrachten geschlossene Geodätische $c=(c(t), 0 \leq t \leq 1)$ auf Flächen. Der Einfachheit halber nehmen wir an, daß die Fläche kompakt ist, obwohl diese Annahme nur für einen Teil unserer Untersuchungen, insbesondere die Existenzsätze, notwendig ist. Die Geodätische $c$ besitzt einen Index; es ist dies die maximale Anzahl von Parametern, mit der man Variationen von $c$ bilden kann, die sämtlich aus kürzeren Kurven bestehen. Dabei sei hier vorausgesetzt, daß die geschlossene Geodätische $c$ nicht-entartet ist.

Die geschlossene Geodätische $c$ bestimmt eine periodische Bahn für den geodätischen Fluß auf dem Tangentialbündel der Fläche. Bezeichne mit $P_{c}$ oder einfach $P$ die (lineare) Poincaré-Abbildung dieser Bahn. $P$

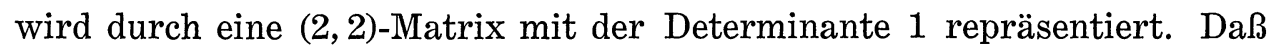
$c$ nicht-entartet ist, ist gleichwertig damit, daß 1 nicht Eigenwert von $P_{c}$ ist. Eine nicht-entartete Geodätische $c$ heißt elliptisch oder hyperbolisch, jenachdem die Eigenwerte von $P_{c}$ den Betrag 1 oder $\neq 1$ besitzen.

Sei $k$ die Anzahl der konjugierten Punkte auf $c$. $k$ ist also die Anzahl der Nullstellen eines Jacobifeldes $Y(\mathrm{t})$ längs $c(t)$ mit $Y(0)=0$, $\nabla Y(0) \neq 0$ im Intervall ]0,1[. Wie wir unten sehen werden, ist für elliptische Geodätische $k$ unabhängig von der Wahl des Anfangspunktes auf $c$, während bei hyperbolischen Geodätischen dies nicht zu gelten braucht.

Schließlich unterscheiden wir bei einer geschlossenen Geodätischen $c$ noch, ob sie orientierbar ist oder nicht. Im ersten Falle ist der durch $c$ bestimmte Flächenstreifen ein Zylinderband, im zweiten ein Möbiusband. Es ist klar, daß es auf einer orientierbaren Fläche nur orientierbare geschlossene Geodätische gibt. Dagegen existieren auf einer nicht-orientierbaren Fläche auch nicht-orientierbare geschlossene Geodätische.

Received December 8, 1976.

* Die Arbeit entstand während eines Gastaufenthaltes an der für ihre geometrische Tradition bekannten Universität Graz. 
2. Für elliptische Geodätische beweisen wir den folgenden

SATz 1. Sei c eine nicht-entartete periodische elliptische Geodätische auf einer Fläche. Sei $k$ die Anzahl der konjugierten Punkte auf $c$.

Wenn c orientierbar ist, so hat $c$ den Index $k+1$ oder $k$, jenachdem ob $k$ gerade oder ungerade ist.

Wenn $c$ nicht orientierbar ist, so hat $c$ den Index $k$ oder $k+1$, jenachdem ob $k$ gerade oder ungerade ist.

Folgerung 1.1. Die Anzahl der konjugierten Punkte auf einer nichtentarteten elliptischen Geodätischen ist unabhängig von der Wahl des Anfangspunktes.

FOLGERUNG 1.2. Auf einer orientierbaren Fläche haben nicht-entartete elliptische Geodätische stets ungeraden Index.

Wir erinnern an die bekannte Tatsache, daß jede Klasse frei-homotoper geschlossener Kurven, die nicht die Punktkurven enthält, auch Kurven minimaler Länge enthält; solche sind (bis auf die Parameterisierung) geschlossene Geodätische vom Index 0. Damit formulieren wir

FolgERUNG 1.3. Sei c eine geschlossene Geodätische minimaler Länge in einer Klasse frei-homotoper Kurven. Wenn c orientierbar ist (insbesondere also, wenn die Fläche orientierbar ist), so ist c hyperbolisch oder entartet. Sämtliche Überlagerungen von c haben den Index 0.

Insbesondere besitzt somit jede orientierbare Fläche vom Geschlecht $>0$ geschlossene Geodätische vom schwach-hyperbolischen Typ. Dies gilt aber ganz allgemein für geschlossene Flächen:

Folgerung 1.4. Auf jeder geschlossenen Fläche existieren geschlossene Geodätische, die entweder entartet oder hyperbolisch sind.

3. Bemerkungen. 1. Beispiele für elliptische und hyperbolische geschlossene Geodätische liefern die Ellipsen auf dem 3-achsigen Ellipsoid, vgl. auch Morse [Mor 2], p. 314.

2. Sei $M$ eine orientierbare geschlossene Fläche vom Geschlecht $\geq 1$. Bezeichne mit $\tilde{M}$ die universelle Überlagerung von $M$ und mit $\gamma$ ein Element $\neq 1$ der Gruppe $\Gamma$ dar Decktransformationen, die auf $\tilde{M}$ freiisometrisch operiert, so daß $M=\tilde{M} / \Gamma$. Es gibt dann auf $\tilde{M}$ vollständige Geodätische $\tilde{c}$, die invariant sind unter $\gamma$ und bei denen der Abstand 
zwischen $\tilde{c}(0)$ und $\gamma \tilde{c}(0)$ minimal ist:

$$
d(\tilde{c}(0), \gamma \tilde{c}(0)) \leq d(\tilde{p}, \gamma \tilde{p}), \quad \text { für alle } \tilde{p} \in \tilde{M},
$$

vgl. [K 4]. Die Folgerung 1.3 impliziert, daß auch für alle Potenzen $\gamma^{q}$ von $\gamma$ gilt: $d\left(\tilde{c}(0), \gamma^{q} \tilde{c}(0)\right) \leq d\left(\tilde{p}, \gamma^{q} \tilde{p}\right)$. Damit gehören diese $\tilde{c}$ zu den von Morse [Mor 1], p. 53 gefundenen periodischen Geodätischen ("boundary periodic geodesics").

Während gemäß Folgerung 1.4 auf einer geschlossenen Fläche stets schwach-hyperbolische Geodätische existieren, ist die Existenz von schwachelliptischen Geodätischen sicherlich vom Geschlecht der Fläche abhängig: Jedenfalls sind auf einer Fläche negativer Krümmung (was für orientierbare Flächen bekanntlich impliziert, daß das Geschlecht $>1$ ist) alle geschlossenen Geodätischen hyperbolisch, vgl. [K 3]. Dagegen scheint es sicher, daß es auf dem Torus und der Sphäre bei beliebiger Metrik stets schwachelliptische Geodätische gibt. Im Falle der Sphäre stellen wir uns einen Beweis dieser Behauptung folgendermaßen vor: Betrachte Deformationen der Metrik des 3-achsigen Ellipsoids. Wir nehmen an, daß eine beliebige Metrik auf $S^{2}$ approximiert werden kann durch solche Deformationen, bei denen nur endlich viele der generischen Bifurkationen von Meyer [Me] vorkommen. Da wir aus Satz 1 wissen, daß elliptische Geodätische ungeraden Index besitzen, und da überdies der Raum der geschlossenen Kurven auf $S^{2}$ den bekannten 1-Zykel bestehend aus den zu einem Großkreis parallelen Kreisen enthält, der Anlaß gibt zu einer geschlossenen Geodätischen vom Index 1, so kann man die behauptete Existenz einer elliptischen Geodätischen erschließen. Der Index dieser Geodätischen kann allerdings nicht a priori nach oben abgeschätzt werden.

Schon Poincaré [Po] hat die Existenz einer elliptischen Geodätischen auf einer analytischen konvexen Fläche behauptet. Allerdings scheint uns sein Beweis unvollständig zu sein. In jedem Falle ist die Existenz einer elliptischen Geodätischen von großem Interesse: Birkhoff [B], p. 186, hat gezeigt, daß solche vom allgemeinen stabilen Typ (und solche existieren generisch, wenn es überhaupt stets schwach-elliptisch geschlossene Geodätische gibt) die Existenz von unendlich vielen weiteren geschlossenen Geodätischen implizieren. Nach Moser [Mos] besitzt die abgeschlossene Hülle des Raumes der periodischen Anfangsrichtungen dann sogar ein positives Maß.

4. Wir kommen jetzt zum Beweis von Satz 1. Bezeichne mit $Y(t)$ 
ein Jacobifeld längs $c(t)$ mit $Y(0),|\nabla Y(0)|=1$. Setze $\dot{c}(t) /|\dot{c}(t)|=E_{0}(t)$, $\nabla Y(0)=E_{1}(0)$. Definiere $E_{1}\left(t_{0}\right), 0 \leq t_{0} \leq 1$, durch Parallelverschiebung von $E_{1}(0)$ längs $c \mid\left[0, t_{0}\right]$. Dann ist $E_{1}(1)= \pm E_{1}(0)$, jenachdem ob $c$ orientierbar ist oder nicht.

Wir betrachten die durch $\phi_{t} \dot{c}(0)=\dot{c}(t), 0 \leq t \leq 1$, gegebene periodische Bahn des geodätischen Flusses auf TM. Da wir von $c$ nicht vorausgesetzt hatten, daß es prim ist, kann es ein ganzes $q>1$ geben so, daß $\dot{c}(1 / q)=\dot{c}(0)$. Das heißt, 1 braucht nicht die kleinste Periode der Bahn $\phi_{t} \dot{c}(0), t \in \boldsymbol{R}, \mathrm{zu}$ sein.

Längs $\phi_{t} \dot{c}(0)$ wählen wir ein begleitendes orthonormales 2-Bein von der Gestalt $\tilde{E}_{1}(t)=\left(E_{1}(t), 0\right)=$ horizontaler Vektor über $E_{1}(t)$ und $\tilde{E}_{2}(t)=$ $\left(0, E_{1}(t)\right)=$ vertikaler Vektor über $E_{1}(t)$ in $\phi_{t} \dot{c}(0)$. Die Vektoren $\tilde{E}_{0}(t)=$ $\left(E_{0}(t), 0\right), \tilde{E}_{1}(t), \tilde{E}_{2}(t)$ spannen den Tangentialraum an die 3-dimensionale Untermannigfaltigkeit $\{X \in T M ;|X|=|\dot{c}|\}$ von $T M$ auf; diese Untermannigfaltigkeit enthält die periodische Bahn $\phi_{t} \dot{c}(0)$.

Offenbar gilt $\tilde{E}_{i}(1)= \pm \tilde{E}_{i}(0), i=1,2$, jenachdem ob $c$ orientierbar ist oder nicht.

Einem Jacobifeld $X(t)$ längs $c(t)$ entspricht das $\phi_{t}$-invariante Vektorfeld $\tilde{X}(t)=(X(t), \nabla X(t))$, wo $X(t), \nabla X(t)$ die horizontale bzw. vertikale Komponente von $\tilde{X}(t)$ sind. Wenn wir also $X(t)=x(t) E_{1}(t)$ schreiben, so ist

$$
\tilde{X}(t)=x(t) \tilde{E}_{1}(t)+\dot{x}(t) \tilde{E}_{2}(t) .
$$

Seien $\tilde{X}(t), \tilde{Y}(t)$ das Fundamentalsystem von Jacobifeldern, d.h., $(\tilde{X}(0), \tilde{Y}(0))=\left(\tilde{E}_{1}(0), \tilde{E}_{2}(0)\right)$. Die Poincaré-Abbildung $P_{c}$ ist gegeben durch

$$
\left(\tilde{E}_{1}(0), \tilde{E}_{2}(0)\right) \longmapsto(\tilde{X}(1), \tilde{Y}(1)) .
$$

Die Matrixdarstellung von $P=P_{c}$ lautet also

$$
\left(\begin{array}{ll}
a & b \\
c & d
\end{array}\right)= \pm\left(\begin{array}{ll}
x(1) & y(1) \\
\dot{x}(1) & \dot{y}(1)
\end{array}\right)
$$

mit dem + oder - Zeichen, jenachdem ob $c$ orientierbar ist oder nicht.

5. Wir definieren mit Hilfe von $\tilde{Y}(t)$ das $\phi_{t}$-invariante Vektorfeld $\tilde{Z}(t)$ durch

$$
\tilde{Z}(t)=(P-\mathrm{id})^{-1} \tilde{Y}(t) .
$$

Für jede Nullstelle $t_{0}=0<\ldots<t_{k}<1$ von $Y(t)$ erklären wir ein 
periodisches Vektorfeld $\zeta_{j}(t)$ längs $c(t)$ durch

$$
\zeta_{j}(t)= \begin{cases}Z(t+1) & 0 \leq t \leq t_{j} \\ Z(t) & t_{j} \leq t \leq 1 .\end{cases}
$$

Also

$$
\zeta_{j}\left(t_{j}^{-}\right)-\zeta_{j}\left(t_{j}^{+}\right)=0, \quad \nabla \zeta_{j}\left(t_{j}^{-}\right)-\nabla \zeta_{j}\left(t_{j}^{+}\right)=\nabla Y\left(t_{j}\right) .
$$

Sei $D^{2} E(c)$ die Indexform auf dem Raum $T_{c} \Lambda M$ der periodischen $H^{1}$ Vektorfelder längs $c$. Die Bezeichnungen sollen daran erinnern, daß dies die Hessische der differenzierbaren Funktion $E: \Lambda M \rightarrow R$ auf dem Raum $A M$ der $H^{1}$-Abbildungen $c^{*}: S \rightarrow M$ ist, berechnet in dem kritischen Punkt $c$, vgl. [K 3]. Es ist also, für $i \leq j$,

$$
\begin{aligned}
D^{2} E(c)\left(\zeta_{i}, \zeta_{j}\right)= & \int_{S}\left\langle\nabla \zeta_{i}, \nabla \zeta_{j}\right\rangle(t)-\left\langle R\left(\zeta_{i}, \dot{c}, \dot{c}\right), \zeta_{j}\right\rangle(t) d t \\
= & -\int_{S}\left\langle\nabla^{2} \zeta_{i}+R\left(\zeta_{i}, \dot{c}, \dot{c}\right), \zeta_{j}\right\rangle(t) d t \\
& +\left\langle\nabla \zeta_{i}\left(t_{i}^{-}\right)-\nabla \zeta_{i}\left(t_{i}^{+}\right), \zeta_{j}\left(t_{i}\right)\right\rangle \\
= & \left\langle\nabla Y\left(t_{i}\right), Z\left(t_{i}\right)\right\rangle=\langle\nabla Y(0), Z(0)\rangle=z(0),
\end{aligned}
$$

wobei wir in der letzten Gleichung die Unabhängigkeit von $t$ des Ausdrucks $\langle\nabla Y(t), Z(t)\rangle-\langle Y(t), \nabla Z(t)\rangle$ benutzt haben.

Wir bestimmen $z(0)$ aus der Gleichung

$$
(P-\mathrm{id}) \tilde{Z}(0)=\tilde{Y}(0)
$$

und finden

$$
z(0)=-b /(2-a-d), \quad b= \pm y(1),
$$

mit dem + oder - Zeichen, jenachdem ob $c$ orientierbar ist oder nicht. Da $c$ elliptisch ist, ist Spur $P_{c}<2$, also $2-a-d>0$.

Die $k+1$ Vektoren $\zeta_{j}, 0 \leq j \leq k$, sind linear unabhängig. Sie spannen einen Unterraum $U$ von $T_{c} \Lambda M$ auf. Mit $U^{\prime}$ bezeichnen wir den von den $k$ linear unabhängigen Vektoren $\eta_{j}=\zeta_{j}-\zeta_{0}, 1 \leq j \leq k$, aufgespannten Raum.

Aus den vorstehenden Rechnungen ergibt sich: $D^{2} E(c) \mid U^{\prime} \equiv 0$. Falls $y(1) \geq 0$ und $c$ orientierbar, so ist sogar $D^{2} E(c) \mid U \leq 0$ (negativ semidefinit). Falls $y(1) \leq 0$ und $c$ nicht-orientierbar, so gilt das gleiche.

Beachte nun, daß der Index einer nicht-entarteten geschlossenen Geodätischen $c$ mindestens so groß ist wie die Dimension eines Unterraums 
$V$ von $T_{c} \Lambda M$ mit $D^{2} E(c) \mid V \leq 0$. Wir haben also gezeigt: Falls $c$ orientierbar (nicht-orientierbar) und $y(1) \geq 0(y(1) \leq 0)$, so ist Index $c \geq k+1$. Falls dagegen $y(1)<0(y(1)>0)$, so ist Index $c \geq k$.

6. Sei weiterhin $c$ eine nicht-entartete elliptische geschlossene Geodätische. Wenn $k$ die Anzahl der konjugierten Punkte auf $c$ ist, so bedeutet dies: Das Vektorfeld $\tilde{Y}(t)=(Y(t), \nabla Y(t))$ trifft im Intervall ]0,1] $k$ Mal die vertikale Gerade $\left\{\tilde{E}_{2}(t)\right\}$. Wenn $\tilde{Y}\left(t_{0}\right) \in\left\{\tilde{E}_{2}\left(t_{0}\right)\right\}$, d.h., $\tilde{Y}\left(t_{0}\right)=$ $\dot{y}\left(t_{0}\right) \tilde{E}_{2}\left(t_{0}\right)$, so $\dot{\tilde{Y}}\left(t_{0}\right)=\dot{y}\left(t_{0}\right) \tilde{E}_{1}\left(t_{0}\right)$, wie aus den Jacobigleichungen folgt. Das heißt, der Schnitt von $\tilde{Y}(t)$ mit der vertikalen Geraden verläuft stets transversal im Uhrzeigersinn.

Sei $c$ orientierbar; dann ist $(y(1), \dot{y}(1)) \neq(0,1)$. Wenn $k$ ungerade ist, so muß also $\tilde{Y}(1)$ in der linken Halbebene $\left\{x_{1} \tilde{E}_{1}(0)+x_{2} \tilde{E}_{2}(0) ; x_{1}<0\right\}$ liegen, d.h. $y(1)<0$. Wenn dagegen $k$ gerade ist, so ist $y(1) \geq 0$-beachte, daß $(y(1), \dot{y}(1))=(0,-1)$ zulässig ist.

Sei $c$ nicht-orientierbar; dann ist $(y(1), \dot{y}(1)) \neq(0,-1)$. Wenn $k$ ungerade ist, so gilt $y(1) \leq 0$; für gerades $k$ ist dagegen $y(1)>0$.

Damit haben wir gezeigt, daß der Index von $c$ mindestens so groß ist, wie in dem Satz 1 behauptet wird. Es bleibt nachzuweisen, daß der Index nicht größer als die angegebenen Zahlen sein kann. Wie man sich leicht überlegt, läuft dies darauf hinaus nachzuweisen, daß die Unterräume $U$ bzw. $U^{\prime}$ nicht um ein Element $\xi \in T_{c} \Lambda, \xi \neq 0$, erweitert werden können, für das gilt:

(i) $D^{2} E(\xi, \xi) \leq 0$

(ii) $D^{2} E\left(\zeta_{j}, \xi\right)=0,0 \leq j \leq k$.

Die Bedingung (ii) bedeutet:

$$
0=D^{2} E(c)\left(\zeta_{j}, \xi\right)=\left\langle\nabla Y\left(t_{j}\right), \xi\left(t_{j}\right)\right\rangle .
$$

Das heißt, $\xi(t)$ verschwindet an den Nullstellen von $Y(t)$. Wir können also annehmen: $\xi(t)=w(t) Y(t)$ mit differenzierbarem $w(t)$. Eine elementare Rechnung, bei $\operatorname{der} \xi(0)=\xi(1)=0$ verwendet werden, liefert mit (i) :

$$
\begin{aligned}
0 \geq D^{2} E(\xi, \xi) & =\int_{0}^{1} \dot{w}(t)^{2}|Y(t)|^{2} d t+\int_{0}^{1} \frac{d}{d t}\langle w \nabla Y, w Y\rangle(t) d t \\
& =\int_{0}^{1} \dot{w}(t)^{2}|Y(t)|^{2} d t .
\end{aligned}
$$

Also $\dot{w}(t)=0, w(t)=w_{0}=$ const. Aus $w_{0} \tilde{Y}(0)=(\xi(0), \dot{\xi}(0))=(\xi(1), \xi(1))=$ $w_{0} \tilde{Y}(1)$ folgt $\xi=0$. Damit ist Satz 1 bewiesen. 
7. Die Folgerungen 1.1 und 1.2 sind unmittelbar klar. Zum Beweis von 1.3 bemerken wir: Da $c$ minimale Länge besitzt, ist Index $c=0$. Da $c$ orientierbar ist, muß nach Satz $1 c$ entweder hyperbolisch nicht-entartet sein oder entartet. Im ersten Falle folgt aus dem Indexsatz für hyperbolische Geodätische, siehe Folgerung 2.1 unten oder [K 1]: Index $c^{q}=q \cdot$ Index $c=0$. Im zweiten Fall gibt es ein periodisches Jacobifeld $\tilde{Y}(t)=(Y(t), \nabla Y(t))$ längs $c(t)$. Wegen Index $c=0$ und $c$ orientierbar kann $\tilde{Y}(t)$ für kein $t$ die vertikale Gerade $\left\{\tilde{E}_{2}(t)\right\}$ treffen, d.h., $Y(t) \neq 0$ für alle $t$. Sei $\xi=\xi(t)$ periodisches Vektorfeld längs $c^{q}(t)=c(q t)$. Dann gilt $\xi(t)=w(t) Y_{q}(t)$ mit $Y_{q}(t)=Y(q t)$. Wie oben liefert die Bedingung $0 \geq D^{2} E\left(c^{q}\right)(\xi, \xi): \xi=w_{0} Y_{q}(t)$, d.h., $\xi \in$ Nullraum $D^{2} E\left(c^{q}\right)$, d.h., In$\operatorname{dex} c^{q}=0$.

Die Behauptung aus Folgerung 1.4 ist wegen 1.3 jedenfalls richtig für orientierbare Flächen vom Geschlecht $>0$. Wenn die Fläche vom Geschlecht Null, also vom Typ der Sphäre $S^{2}$ ist, so gibt es eine geschlossene Geodätische $c$, an der ein Zykel der Dimension 2 hängen bleibt, vgl. Lusternik und Schnirelmann [LS] order [K 3], Chap. II. Wenn c nicht-entartet ist, so ist Index $c=2$, nach Satz 1 ist daher $c$ hyperbolisch. Wenn die betrachtete geschlossene Fläche $M$ nicht orientierbar ist, so gehen wir zur orientierbaren 2-fachen Überlagerung über; eine hierauf gefundene schwach-hyperbolische Geodätische projiziert sich auf eine ebensolche auf $M$.

8. Wir ergänzen unseren Satz 1 über den Index einer elliptischen geschlossenen Geodätischen durch einen Satz über den Index einer hyperbolischen geschlossenen Geodätischen. Unser Satz kann aus einem Satz in [K 1] über hyperbolische geschlossene Geodätische auf Mannigfaltigkeiten beliebiger Dimension abgelesen werden.

Zunächst bemerken wir, daß eine nicht-entartete hyperbolische Geodätische $c=(c(t), 0 \leq t \leq 1)$ auf einer Fläche ein sogenanntes stabiles Jacobifeld $Y_{s}(t)$ besitzt. Dies ist charakterisiert durch die Eigenschaft: $\tilde{Y}_{s}(1)=\rho \tilde{Y}_{s}(0), \operatorname{mit}|\rho|<1, \tilde{Y}_{s}(t)=\left(Y_{s}(t), \nabla Y_{s}(t)\right)$. Vgl. hierzu [K 1], [K 2], [K 3]. Damit gilt nun

SATZ 2. Sei c eine hyperbolische geschlossene Geodätische. Dann ist Index c gleich der Anzahl der Nullstellen des stabilen Jacobifeldes längs $c$.

FOLGERUNG 2.1. Für eine hyperbolische geschlossene Geodätische c ist Index $c^{q}=q \cdot$ Index $c$. 
FolGERUNG 2.2. Für eine hyperbolische geschlossene Geodätische ist der Index gleich der Maximalzahl konjugierter Punkte von $c\left(t_{0}\right)$ in $] t_{0}$, $\left.t_{0}+1\right]$, für alle möglichen Wahlen des Anfangspunktes $t_{0}$. Dieses Maximum wird insbesondere für diejenigen $t_{0} \in[0,1]$ angenommen, in denen das stabile bzw. das unstabile Jacobifeld eine Nullstelle besitzt. Es sind dies gleichzeitig diejenigen $t_{0}$, in dem $c\left(t_{0}+1\right)$ konjugiert ist zu $c\left(t_{0}\right)$.

Folgerung 2.3. Seien $\rho, 1 / \rho,|\rho|<1$, die Eigenwerte der PoincaréAbbildung $P_{c}$ einer hyperbolischen geschlossenen Geodätischen. Dann ist Index c gerade genau denn, wenn entweder $c$ orientierbar ist und $\rho>0$, oder $c$ nicht-orientierbar und $\rho<0$.

9. Zum Beweis von Satz 2 betrachten wir das bis auf einen konstanten Faktor $\neq 0$ festgelegte stabile $\phi_{t}$-invariante Feld $\tilde{Y}_{s}(t)=\left(Y_{s}(t)\right.$, $\left.\nabla Y_{s}(t)\right)$ längs $\phi_{t} \dot{c}(0)$. Falls $Y_{s}(t) \neq 0$ für alle $t$, so ergibt sich genau wie in (7), daß Index $c=0$.

Nimm jetzt an: $Y_{s}\left(t_{0}\right)=0$, d.h., $\tilde{Y}_{s}\left(t_{0}\right)$ gehört zur vertikalen Geraden $\left\{\tilde{E}_{2}\left(t_{0}\right)\right\}$. Sei $k-1$ die Anzahl der Nullstellen von $Y_{s}(t)$ für $t_{0}<t<t_{0}+1$. $k$ ist also die im Satz 2 genannte Zahl. $\ddot{A} h n l i c h$ wie in (5) betrachten wir ein Jacobifeld $Z(t)$, das durch $\tilde{Z}(t)=(P-\mathrm{id})^{-1} \tilde{Y}_{s}(t)$ bestimmt ist. Wegen $P \tilde{Y}_{s}=\rho \tilde{Y}_{s}$ ist offenbar $\tilde{Z}=\tilde{Y}_{s} /(\rho-1)$. Für jede der $k$ Nullstellen $t_{0}<\ldots<t_{k-1}$ von $Y_{s}(t)$ erklären wir ein periodisches Vektorfeld $\zeta_{0}, \cdots$, $\zeta_{k-1}$ wie in (5). Wie dort finden wir für $D^{2} E(c)\left(\zeta_{i}, \zeta_{j}\right)=\left\langle\nabla Y_{s}\left(t_{i}\right), Z\left(t_{i}\right)\right\rangle$ $=0$, da $Z\left(t_{i}\right)=Y_{s}\left(t_{i}\right) /(\rho-1)=0$. Das heißt, die Indexform verschwindet identisch auf dem von den $\zeta_{j}$ aufgespannten $k$-dimensionalen Raum, also Index $c \geq k$. Das $=-$ Zeichen ergibt sich ebenso wie in (6). Damit ist Satz 2 bewiesen.

Die Folgerung 2.1 ergibt sich unmittelbar aus Satz 2: Wenn $c^{q}(t)$ $=c(q t)$ die $q$-fache Überlagerung von $c(t)$ ist, so ist $Y_{s}^{q}(t)=Y_{s}(q t)$ ein stabiles Jacobifeld längs $c(q t)$. Jeder Nullstelle $t_{0}$ von $Y_{s}\left(t_{0}\right)$ entsprechen die $q$ Nullstellen $p t_{0} / q, q=1, \cdots, q$, von $Y_{s}^{q}\left(t_{0}\right)$.

Zum Beweis der Folgerung 2.2 bemerken wir, daß der Sturmsche Satz über die Trennung von Nullstellen linear unabhängiger Lösungen der Jacobigleichung (vgl. [K 4]) impliziert: Wenn $Y_{s}(t) k \geq 0$ Nullstellen in 10,1] besitzt, so ist die Anzahl der konjugierten Punkte von $c\left(t_{0}\right)$ in ]$\left.t_{0}, t_{0}+1\right]$ höchstens gleich $k$. Wenn $Y(t)$ ein nicht-triviales Jacobifeld mit $Y\left(t_{0}\right)=Y\left(t_{0}+1\right)=0$ ist, so ist offenbar $\tilde{Y}=(Y, \nabla Y)$ Eigenvektor von $P$. Das heißt, $\tilde{Y}=\tilde{Y}_{s}$ oder $\tilde{Y}_{u}$, wo $P \tilde{Y}_{u}=(1 / \rho) \tilde{Y}_{u}$, d.h., $Y_{u}$ ist das 
in stabile Jacobifeld. In jedem Falle ist die Anzahl der konjugierten Punkte von $c\left(t_{0}\right)$ in $] t_{0}, t_{0}+1$ ] genau gleich dem Index von $c$. Wenn dagegen $Y\left(t_{0}\right)=0, Y\left(t_{0}+1\right) \neq 0$, so kann die Anzahl der konjugierten Punkte von $c\left(t_{0}\right)$ in ] $t_{0}, t_{0}+1$ [ die Werte $k$ oder $k-1$ annehmen, $k=$ Index.

Beim Beweis der Folgerung 2.3 beschränken wir uns auf den Fall, daß $c$ orientierbar ist. Aus $\tilde{Y}_{s}(1)=\rho \tilde{Y}_{s}(0)$ folgt: Die Anzahl der Schnitte von $\tilde{Y}_{s}(t)$ mit der vertikalen Geraden für $\left.\left.t \in\right] 0,1\right]$ ist gerade oder ungerade, jenachdem ob $\rho>0$ oder $\rho<0$ ist. Im Falle $\rho<0$ gibt es also ein $t_{0}$ mit $Y_{s}\left(t_{0}\right)=0$, und es gibt dann noch eine gerade Anzahl $k-1 \geq 0$ von Nullstellen von $Y_{s}(t)$ in $] t_{0}, t_{0}+1[$. Also Index $c=k$ ungerade. Im Falle $\rho>0$ ist entweder $Y_{s}(t) \neq 0$ für alle $t$, also Index $c=0$. Oder sonst gibt es $t_{0}$ mit $Y_{s}\left(t_{0}\right)=0$ und noch $k-1$ weitere Nullstellen von $Y_{s}(t)$ in ]$t_{0}, t_{0}+1[$. Nach Satz 2 ist dann Index $c=k$ gerade.

10. Als einfache Anwendung von (2.1) beweisen wir noch zum Schluß die Existenz einer geschlossenen elliptischen Geodätischen auf einer konvexen Fläche mit fixpunktfreier Involution. Auf das große Interesse, welches der Existenz elliptischer geschlossener Geodätischer zukommt, hatten wir bereits in Abschnitt (3) hingewiesen.

Satz 3. Sei $M$ eine geschlossene Fläche positiver Krümmung mit einer fixpunktfreien isometrischen Involution $J: M \rightarrow M$. Dann existiert auf $M$ eine schwach-elliptische geschlossene Geodätische vom Index 1.

Zum Beweis betrachten wir auf der projektiven Ebene $M / J$ eine geschlossene Geodätische $c_{0}$ minimaler Länge in der Klasse der nichtnullhomotopen geschlossenen Kurven. $c_{0}$ ist nicht orientierbar. Bezeichne mit $c$ die zweifache Überlagerung von $c_{0}$, betrachtet als geschlossene Geodätische auf $M$. Wir wollen zeigen, daß $c$ entartet ist oder elliptisch und vom Index 1.

In der Tat, da die Krümmung auf $M$ positiv ist, ist Index $c>0$. Andererseits ist Index $c_{0}=0$. Wegen (2.1) kann also $c=c_{0}^{2}$ nicht hyperbolisch sein.

Betrachten wir zunächst den Fall, daß $c$ nicht-entartet ist. Da $c_{0}$ den Index 0 hat, enthält es keine konjugierten Punkte im Innern. Also kann auch $c$ höchstens einen konjugierten Punkt im Innern besitzen. Aus Satz 1 folgt damit: Index $c=1$.

Wenn dagegen $c$ entartet ist, so ist auch $c_{0}$ entartet: Den Jacobifel- 
dern $\tilde{Y}_{0}(t)$ längs $c_{0}(t)$ entsprechen die Jacobifelder $\tilde{Y}(t)=\tilde{Y}_{0}(2 t)$ längs $c(t)$ $=c_{0}(2 t)$. Speziell gibt es also $\tilde{X}_{0}(t)=x_{0}(t) \tilde{E}_{1}(t)+\dot{x}_{0}(t) \tilde{E}_{2}(t) \operatorname{mit}\left(x_{0}(1), \dot{x}_{0}(1)\right)$ $=-\left(x_{0}(0), \dot{x}_{0}(0)\right)$. $\quad x_{0}(t)$ besitzt also eine Nullstelle, und wir können annehmen, daß diese bei 0 liegt.

Für das Jacobifeld $\tilde{X}(t)=\tilde{X}_{0}(2 t)$ längs $c(t)$ gilt also: $X(0)=X(1 / 2)$ $=0$. Wenn wir das periodische Vektorfeld $\zeta$ längs $c$ erklären durch

$$
\zeta(t)= \begin{cases}X(t) ; & 0 \leq t \leq 1 / 2 \\ 0 ; & 1 / 2 \leq t \leq 1\end{cases}
$$

so ist $D^{2} E(c)(\zeta, \zeta)=0, \zeta$ nicht im Nullraum von $D^{2} E(c)$. Also Index $c \geq 1$.

Wegen Index $c_{0}=0$ besitzt $X(t)$ keine weiteren Nullstellen in $[0,1 / 2]$. Ebenso wie in (6) folgt daher für ein periodisches Vektorfeld $\xi$ längs $c$ aus $D^{2} E(c)(\zeta, \xi)=0$ und $D^{2} E(c)(\xi, \xi) \leq 0: \xi(t)=w_{0} X(t)$, d.h., $\xi$ gehört zum Nullraum von $D^{2} E(c)$. Also ist Index $c=1$.

Zusatz bei der Korrektur. Die Resultate dieser Arbeit finden sich z.T. schon bei Hedlund [He].

\section{LITERATUR}

[Bi] G. D. Birkhoff, Dynamical systems. Amer. Math. Soc. Colloq. pub., vol. 9, Amer. Math. Soc., New York, N.Y., 1927. Revised ed., 1966.

[He] G. A. Hedlund, Poincaré's rotation number and Mor'se's type number. Trans. Amer. Math. Soc. 34 (1932), 75-97.

[K 1] W. Klingenberg, Manifolds with geodesic flow of Anosov type. Arn. of Math. 99 (1974), 1-13.

[K 2] - Der Indexsatz für geschlossene Geodätische. Math. Z. 193 (1974), 231256.

[K 3] - Lectures on Closed Geodesies. Springer, Berlin-Heidelberg-New York, 1978.

[K 4] —, Eine Vorlesung über Differentialgeometrie. Springer-Verlag, BerlinGöttingen-Heidelberg, 1973.

[LS] Lusternik und Schnirelmann, Sur le problème de trois géodésiques fermées sur les surfaces de genre O. C. R. Acad. Sci. Paris 189 (1929), 269-271.

[Me] K. Meyer, Generic bifurcation of periodic points. Trans. Amer. Math. Soc. 149 (1970), 95-107.

[Mor 1] M. Morse, A fundamental class of geodesics on any closed surface of genus greater than one. Trans. Amer. Math. Soc. 26 (1924), 25-60.

[Mor 2] - The calculus of variations in the large. Amer. Math. Soc. Colloq. Publ., Vol. 18, Amer. Math. Soc., Providence, R. I., 1934.

[Mos] J. Moser, Stable and random motions in dynamical systems. Ann. of Math. Studies No. 77, Princeton Univ. Press, Princeton, N. J. (1973).

[Pe] H. Poincaré, Sur les lignes géodésiques des surfaces convexes. Trans. Amer. Math. Soc. 6 (1905), 237-274. 